\title{
Target Design and R\&D of J-PARC E06 TREK experiment
}

\section{Youichi Igarashi* KEK}

E-mail: youichi.igarashi@kek.jp

\section{TREK collaboration}

\begin{abstract}
TREK is an experiment to search for violation of time reversal invariance (T-violation) in the kaon decay of $K^{+} \rightarrow \pi^{0} \mu^{+} v$ planned at the Japan Proton Accelerator Research Complex (J-PARC). The active target is one of the primal detector elements of the TREK detector system. Kaons come from the beam line, stop and decay in the target. The active target consists of a bundle of scintillating fiber and detects the vertex point of a decay. We plan to use Avalanche Photo Diode based Multi-Pixel Photon Counter (MPPC) to detect photons come from the scintillating fibers. MPPC has several advantages about the photon detection, however as a semi-conductor detector MPPC is easily damaged by the radiation. We have exposed pions to an MPPC sample to measure the radiation damage of it whether it can be used for the active target, or not. The measurement have done at the TRIUMF MESON HALL M13 beam line. An MPPC sample was exposed $5.18 \times 10^{9}$ pions maximum with $130 \mathrm{MeV} / \mathrm{c}$ energy. We have observed the degradation of the MPPC performance however confirmed that the MPPC works as the photon detector for the TREK active target.
\end{abstract}

2009 KAON International Conference KAON09,

June 09 - 122009

Tsukuba, Japan

${ }^{*}$ Speaker. 


\section{TREK experiment and target}

In the three-body decay of $K_{\mu 3}$, a decay plane is defined in the center-of-mass system. Muon transverse polarization $\left(P_{T}\right)$ is the polarization component perpendicular to this decay plane. Mathematically it can be expressed as a correlation of the three vectors of the muon spin $\left(\sigma_{\mu}\right)$, the pion momentum $\left(p_{\pi}\right)$ and the muon momentum $\left(p_{\mu}\right)$. Since all the involved vectors change sign when time is reversed, this correlation, namely $P_{T}$, has a T-odd character. Under the condition that spurious effects from final state interactions are negligibly small, a non-vanishing value of $P_{T}$ is a clear evidence for $\mathrm{T}$ violation.

The most recent research of $P_{T}$ has been performed at KEK as the E246 experiment. The E246 result was consistent with no $\mathrm{T}$ violation but provided the world best limit of $P_{T}=-0.0017 \pm$ 0.0023 (stat) \pm 0.0011 (syst) and constrained the parameter spaces of several contender models. It was, however, statistics limited, mainly due to insufficient accelerator beam intensity in spite of smaller systematic errors. TREK(Time Reversal Experiment with Kaons) experiment is a successor experiment of the E246. TREK experiment intend to continue the $P_{T}$ experiment further at Japan Proton Accelerator Research Complex (J-PARC) [1] where higher accelerator beam intensity will be available and a higher experimental sensitivity is promised, in order to search for new physics beyond SM. TREK experiment aim for a sensitivity of $\Delta P_{T} \sim 10^{-4}$ [2].

The TREK detector is constructed by upgrading the E246 detector which has been used at the KEK-PS. It consists of a superconducting toroidal magnet, an active kaon stopping target, a CsI(Tl) electromagnetic calorimeter, a charged particle tracking system, which identify the $K_{\mu 3}$ decays, and an active muon polarimeter which measures precisely the transverse component of muon polarization. The toroidal magnet has 12 identical gaps. Muons from the $K_{\mu 3}$ decays are momentum-analyzed by the magnet and the tracking chambers and stopped in the active polarimeter. Two photons from $\pi^{0}$ decay in the $K_{\mu 3}$ decay are detected by the CsI(Tl) calorimeter. The kinematics of the $K_{\mu 3}$ is uniquely determined by the measurements of $\mu^{+}$and $\pi^{0}$. The transverse polarization of muon is determined by observing the azimuthal asymmetry of $\mu^{+}$decay positrons.

The active target of TREK experiment consists of scintillating fibers. Many scintillating fibers are bundled as a cylinder. The scintillation light of each fiber is transported via the optical fiber to the photon sensor. We study to use the clear fiber and the optical fiber with wave length shifter as candidates of the light transport fiber. The size of scintillating fibers has $5 \mathrm{~mm}^{2}$ cross section and the number of them is 492 in current design. The active target figure of the current design is shown in Figure 1.

\section{MPPC radiation damage test}

The most suitable candidate of the photon sensor is Multi Pixel Photon Counter (MPPC) [3]. MPPC is the complex Avalanche Photo-Diode (APD) working on Geiger mode. Several hundred of APD are placed on a sensor chip. MPPC has many advantages to read scintillating fiber, compare with other detectors. Mainly advantages are good photon counting efficiency, working at normal temperature, lower operating voltage (around 70V), precise time resolution and small size. These advantages are suitable to detect the photons from thin optical fibers. And a disadvantage of MPPC which is the small detection face is no issue, because the cross sections of fibers are also small. 


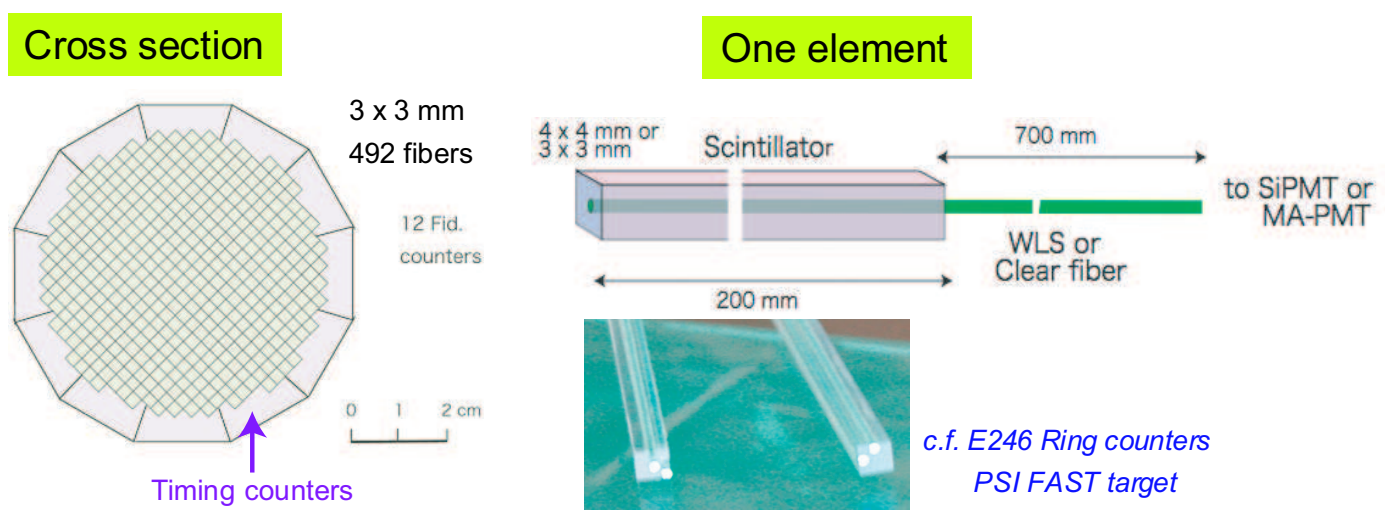

Figure 1: Current design of the active target
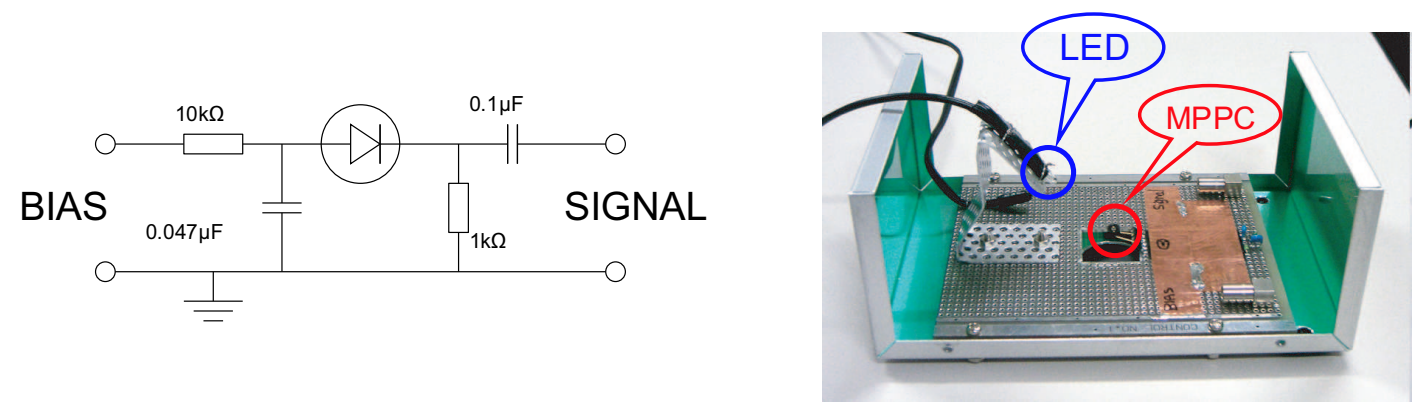

Figure 2: Readout circuit schematic

Figure 3: Picture of the MPPC dark box

However, MPPC has the weakness against the radiation damage, such as other semi-conductor detectors. The radiation damage of MPPC is measured with neutron and positron [4]. However the radiation damage by meson has not yet been measured. The main radiation near the TREK target is background pions. The estimated value of the flux is $\sim 10^{17}$ pion/year. We tested whether the MPPC works under this meson background condition.

\subsection{Setup of the radiation damage test}

The test was done at the TRIUMF MESON HALL M13 beam line. The MPPC sample was exposed to pions with $130 \mathrm{MeV}$ energy. The type of the used MPPC sample was S10362-11-050C. It has 400 pixel cell and a $1 \mathrm{~mm}^{2}$ detection face. The standard operation voltage is $69.74 \mathrm{~V}$. The MPPC was placed in the dark box shown in Figure 3. A thermo sensor and an LED were set in the dark box. The temperature in the dark box was been monitored during the measurement. LED was driven by an LED driver circuit and the light emission was controlled with the timing accuracy better than $10 \mathrm{nsec}$. The circuit to read the signal is shown in Figure. 2. The output signal of the readout circuit was amplified by a PMT amplifier "PHILLIPS SCIENTIFIC Model 777" and read by ADC. The setup of the test and a picture of the setup are shown in Figure 4. The numbers of pions were counted by the coincidence of the scintillation counters S1, S2 and S3. And the number of exposure pions to MPPC was calculated by the beam density profile and the area ratio of the 


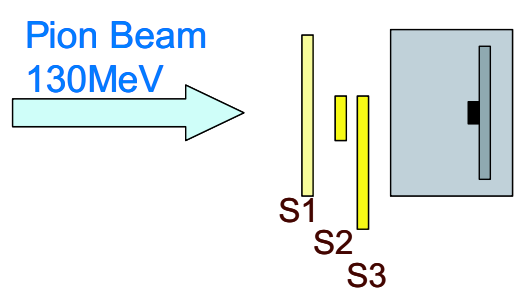

Pion counting counters

Side view of the setup

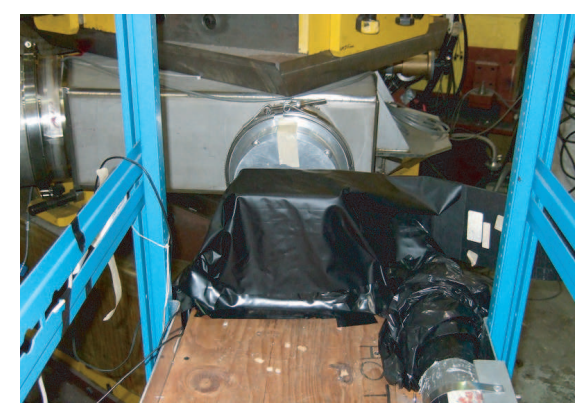

End view of the setup

Figure 4: Setup of the MPPC radiation damage test and a picture of the dark box

Table 1: Exposure summary

\begin{tabular}{|c|c|c|c|c|c|c|c|c|c|c|c|c|}
\hline Exposure & 1 & 2 & 3 & 4 & 5 & Beam stop & 6 & 7 & 8 & 9 & 10 & 11 \\
\hline \hline $\begin{array}{c}\text { S2xS3 } \\
\text { Count }\left(\times 10^{8}\right)\end{array}$ & 126 & 125 & 250 & 259 & 53 & - & 253 & 252 & 503 & 512 & 604 & 907 \\
\hline $\begin{array}{c}\text { Accumulate } \\
\text { Pion }\left(\times 10^{8}\right)\end{array}$ & 1.70 & 3.38 & 6.75 & 10.2 & 11.0 & - & 14.4 & 17.8 & 24.5 & 31.4 & 39.5 & 58.8 \\
\hline
\end{tabular}
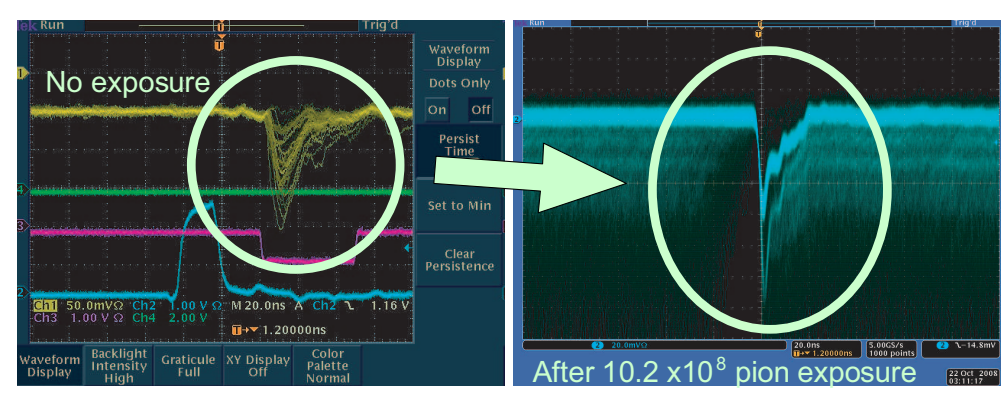

Figure 5: Signal deterioration

counters and the MPPC. The MPPC sample was exposed eleven times, and the beam was stopped during a day after the fifth times exposure. The exposure pion is summarized in Table 1.

\subsection{Effects of the radiation damage}

Figure 5 shows raw signals of the fresh MPPC and the MPPC which was exposed to $10.2 \times 10^{8}$ pions. The operating voltage is same as the standard one. The asynchronous spontaneous signals appeared like lines after exposure. Figure 6 shows the increase of the dark current according to the exposure. Figure 7 shows the current curve by the operating voltage. The charge spectrum of MPPC signals are shown in Figure 8, 9, and 10. Each figure has six plots. The plots show the spectra 1) with no exposure, 2) $3.38 \times 10^{8}$ pions exposure, 3) $10.2 \times 10^{8}$ pions exposure, 4) $14.4 \times 10^{8}$ pions exposure, 5) $31.4 \times 10^{8}$ pions exposure, and 6) $51.8 \times 10^{8}$ pions exposure. Figure 8 shows the spectrum with low photon incidence condition. The separated photon peak is buried in the hot pixels back ground, according to the exposure. However, the width of the each separated photon peak has no large difference. The degradation of the intrinsic gain of MPPC is smaller than $10 \%$. 

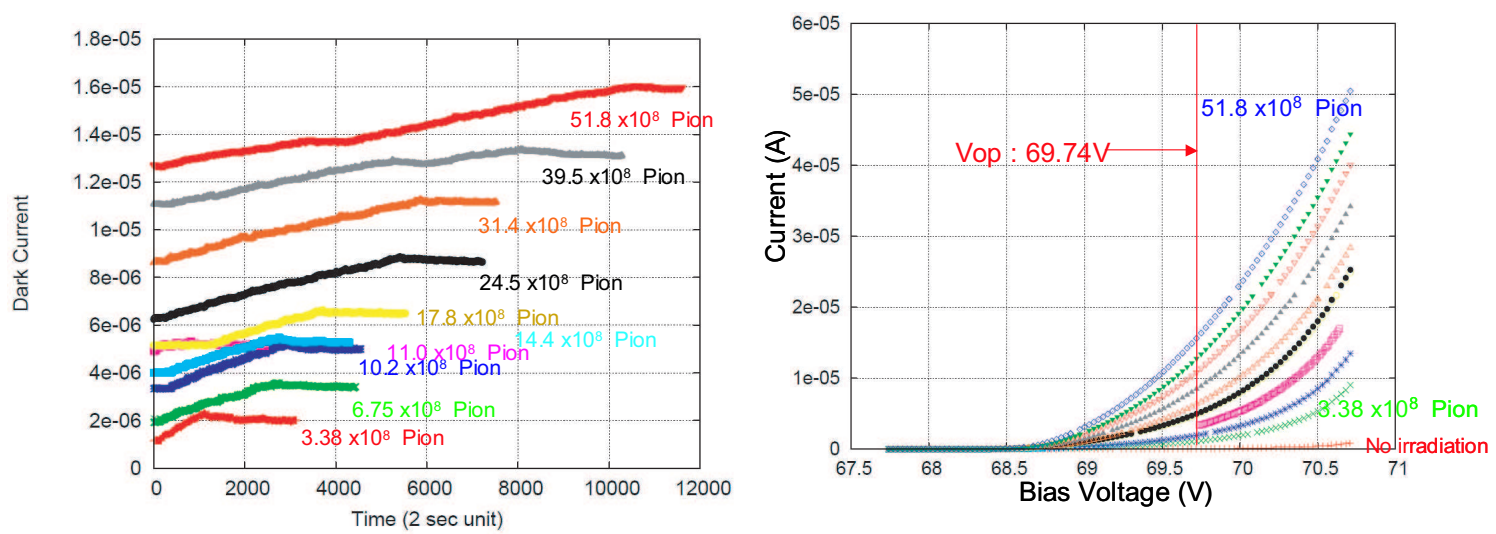

Figure 6: Dark current change during the irradiation

Figure 7: Current Voltage curve, Red line $\left(\mathrm{V}_{\mathrm{op}}\right)$ is the
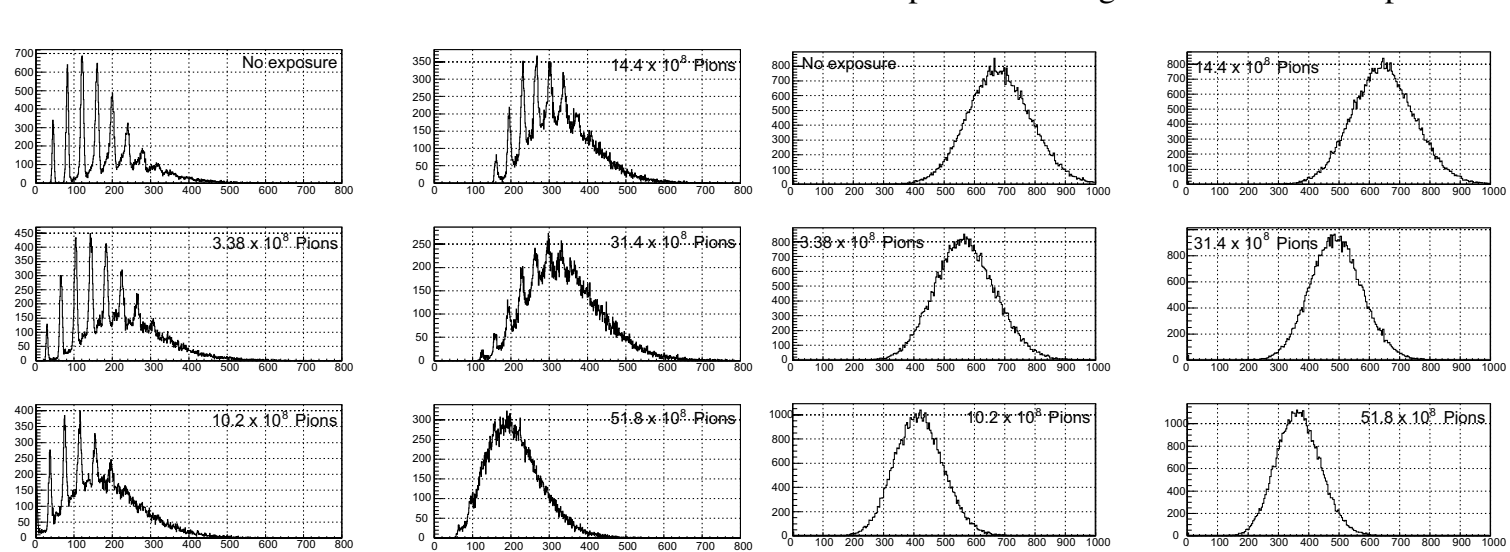

Figure 8: Spectrum deformation of the low photon Figure 9: Spectrum deformation of the 50 photon reregion gion

Figure 9 shows the spectrum with the 50 photons incidence condition. The peak of the spectra shifts to lower side. It is considered that the shift has two reasons. One is the fact that its degradation brought about inactive pixels by the radiation damage. The other was the operation voltage drop by the dark current flowing in the registers of the readout circuit. Figure 10 shows the spectrum with no photon. The strange peak appeared according to the exposure. It is probable that it brought about hot pixels by the radiation damage. Figure 11 shows the 50 photon peak position dependency of the operation voltage. The actual operation voltage of MPPC dropped down by the dark current in the readout circuit registers. The upper region of the "no exposure" MPPC dependence curve in the peak shift brought about the operation voltage drop. The rest in the peak shift, caused by the radiation damage.

\section{Results and conclusion}

We are designing and studying the scintillating fiber based active target for the J-PARC TREK experiment. We plan to use MPPCs as the photon detector of the active target. We 

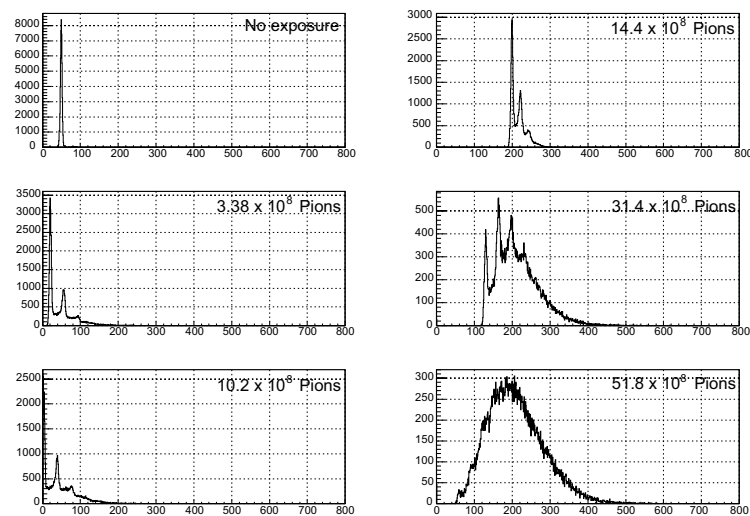

Figure 10: Spectrum deformation of the pedestal sig nal

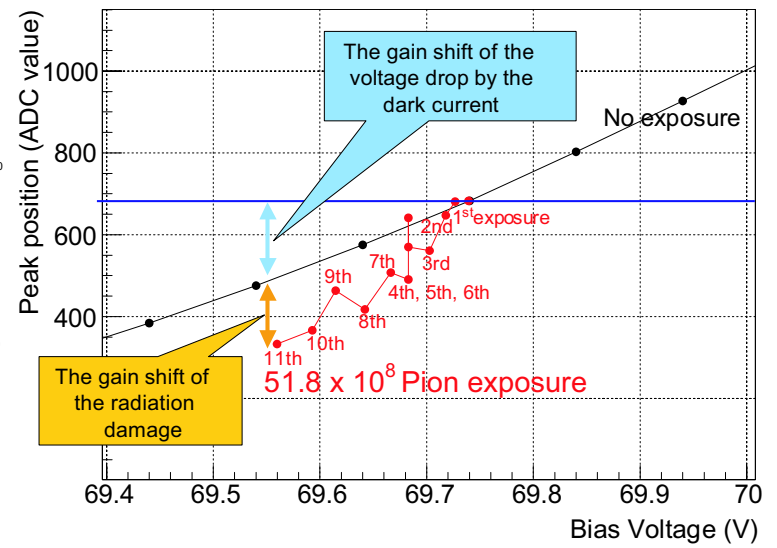

Figure 11: Plot of the peak shift and the effect of dark current

examined how hard an MPPC is against the radiation pion flux, as a part of the active target study. A 400 pixel MPPC sample was exposed $51.8 \times 10^{8} 130 \mathrm{MeV} / \mathrm{c}$ pions $(141 \mathrm{~Gy})$. The estimation of the number of mesons near the TREK target is $10^{7} /$ year $/ \mathrm{mm}^{2}$ The figures show the deterioration of signals and spectra with increasing exposure. Regarding the peak of the 50 photons measurements, the shift to lower direction was observed. 53\% of the peak shift could be attributed to the voltage drop by the register of the readout circuit and the left of $47 \%$ could be concluded due to the radiation damage. Dark current measurements indicate the number of hot pixels increase with radiation damage. MPPC can be used as the readout element of the TREK target since the deterioration level observed in this test experiment is much higher.

\section{References}

[1] The Joint Project Team of JAERI and KEK, "The Joint Project for High-Intensity Proton Accelerators", KEK-PREPRINT-1999-4, Jul 1999

[2] J. Imazato et al., "Measurement of T-violating Transverse Muon Polarization in $K^{+} \rightarrow \pi^{0} \mu^{+} v$ Decays", Proposal for J-PARC $50 \mathrm{GeV}$ Proton Synchrotron, P06, Apr 2006

[3] Hamamatsu Photonics K.K., "MPPC Multi-Pixel Photon Counter", [Online]. Available: http://jp.hamamatsu.com/resources/products/ssd/eng/html/mppc_e/pdf/mppc_kapd9003e02.pdf, May 2009

[4] T. Matsumura et al., "Effects of radiation damage caused by proton irradiation on Multi-Pixel Photon Counters (MPPCs)", Nucl.Instrum.Meth.A 603 (2009), 301-308 